\title{
The Effect of Rest Interval Length on Sustainability of Repetitions during Resistance Training for Front Femoris Muscles
}

\section{* PhD Amr H. Tammam \\ ** PhD Enas M. Hashem}

Abstract:

There is a lack of research that investigate the relationship between rest interval length and the sustainability of repetitions when training with loads designed to develop muscular endurance for similar muscle groups. So this study aimed to compare the effect of 3 different rest interval lengths [1min (RIL1), 2-min (RIL2), and 3-minute (RIL3)] on the sustainability of repetitions for front Femoris muscles (squat, leg press, and knee extension) over 5 consecutive sets performed with a 15 repetition maximum (15RM) load. Fourteen experienced, resistance-trained males volunteered to participate in the study (age, $25.6 \pm 4.1 \mathrm{yr}$; weight, $76.8 \pm 7.1 \mathrm{~kg}$; height, $174.2 \pm 2.43 \mathrm{~cm})$. All subjects were tested weekly over a period of 4 weeks. During the 1st testing session, (15RM) - load for 3 front Femoris muscles exercises performed in a set manner were determined for each subject. During the next 3 testing sessions, 5 consecutive sets of the exercises were performed to voluntary exhaustion. The 3 testing sessions differed only in the length of rest interval between sets and exercises RIL1, RIL2, or RIL3 with a random order to testing sessions. For all exercises, the results showed results demonstrate significantly decline in repetitions completed between the first set and each subsequent set thereafter ( $\mathrm{P} \square 0.05$ ), irrespective of the rest condition, and results demonstrate significant difference in the total repetitions between RIL1 and RIL3 and between RIL2 and RIL3 (P $\square$ 0.05). However, the total of repetitions was not significantly different between RIL1 and RIL2 (P $\square$ 0.05). So when training for similar muscle groups (Front Femoris Muscles); the rest interval length must be long enough $(>=3$ minute) to sustain a high number of repetitions over consecutive sets, or decrease the intensity to sustain repetitions.

Key words: rest interval length, resistance training, multiple exercises, muscle groups, strength training, recovery

"Department of Sports Training, Faculty of Physical Education, Tanta University, Egypt

"Department of Physical Education and Sport Science, Faculty of Education and Arts, Northern Border University, Saudi Arabia Assiut Journal For Sport Science Arts 


\section{Introduction:}

Resistance training can increase maximal strength, hypertrophy, power, and localized muscular endurance. The prescriptive variables are numerous, and may include: type of muscle contractions, rest intervals between sets and exercises, frequency, velocity of movement, number of sets and repetitions, load or intensity, and exercise order. All of these variables can be manipulated to meet specific training goals and address individual needs (Baechle \& Earle, 2000; W. J. Kraemer et al., 2002; Weiss, 1991). Over the last 20 years, resistance exercise research has focused on the intensity and repetition ranges that produce the greatest strength increases in different populations (Baechle \& Earle, 2000; W. J. Kraemer et al., 2002; Rhea, 2003). However, one training variable that has received relatively little attention is the rest interval between sets (Willardson, 2006). Including sufficient rest between sets is essential, particularly when the goal is maximal strength development (Weiss, 1991).

Rest intervals between sets appear to be an important variable that can be manipulated to fit the goal of resistance training and can directly affect training volume and fatigue by altering endocrinal and metabolic responses as well as the performance and completion of subsequent sets (Fleck and Kraemer, 2004). it is well known that higher training intensities $(\geq 11 \mathrm{RM})$ lead to greater muscle strength and hypertrophy whereas lower intensities $(\leq 20 \mathrm{RM})$ are related to greater muscle endurance adaptations (Campos et al., 2002). When the training goal is maximal strength development, multiple sets per muscle group were superior to single sets. However, the superiority of performing multiple sets per muscle group may depend on the ability to sustain consistent repetitions over consecutive sets (Robinson et al., 1995). The ability to sustain consistent repetitions is largely dependent on the length of the rest interval between sets (W. J. Kraemer, 1997; Matuszak, Fry, Weiss, Irelamd, \& Mcknight, 2003; Richmond \& Godard, 2004; Todd, Sjuts, Krosch, Conley, \& Evetovich, 2001;

Assiut Journal For Sport Science Arts 
Weir, Wagner, \& Housh, 1994; Willardson \& Burkett, 2005, 2006). The length of rest interval must be sufficient to recover energy sources (e.g., adenosine triphosphate [ATP] and phosphocreatine [PCr]), clear fatigue producing substances (e.g., H_ions), and restore force production (Baechle \& Earle, 2000; W. J. Kraemer et al., 2002; Sahlin \& Ren, 1989; Weiss, 1991).

The effect of rest between sets on exercise volume in single exercises has been investigated by some previous studies demonstrated significant differences in repetition performance and the exercise volume completed (W. J. Kraemer, 1997; Larson Jr \& Potteiger, 1997; Rahimi, 2005; Richmond \& Godard, 2004; Willardson \& Burkett, 2005). Additionally, (Ratamess et al., 2007) compared the differences in workout volume (resistance $\mathrm{x}$ repetitions per set) over five sets of the bench press exercise when performed at two different intensities (i.e. $75 \%$ and $85 \%$ of a 1RM) and with five different rest intervals between sets (i.e. 30 seconds, $1,2,3$, and 5 minutes). The findings demonstrated that irrespective of the intensity, the workout volume significantly decreased with each set in succession over five sets when 30-second and 1-min rest intervals were used. The workout volume was maintained over two sets for 2 minutes, three sets for 3 minutes, and four sets for 5 minutes. Consequently, the authors recommended that if more than 2 to 3 sets of an exercise are performed, then at least 2 minutes of rest might be needed to minimize load reductions and maintain repetition performance for the sets performed at the end of a workout.

However, a limitation of (Ratamess et al., 2007) and similarly designed studies (W. J. Kraemer, 1997; Mirzaei, Nia, \& Saberi, 2008; Rahimi, 2005; Richmond \& Godard, 2004; Willardson \& Burkett, 2005, 2006) was the examination of a single exercise, when typical resistance sessions consist of multiple exercises for the same muscle groups (W. J. Kraemer et al., 2002; W. J. Kraemer \& Ratamess, 2005). However, there is a great need for further research to compare the sustainability of repetitions in multiple exercises over an 
entire resistance training session for the same muscle groups with different rest intervals between sets. According to our knowledge, the effect of 1- or 2-minute or 3 -minute rest interval on the sustainability of repetition for front Femoris muscles group with 15RM load has not been reported, Therefore, the purpose of the current study was to compare the effect of 3 different rest interval lengths [1-min (RIL1), 2-min (RIL2), and 3-minute (RIL3)] between sets and exercise on the sustainability of repetitions (number of repetitions per set, total repetitions) over 5 consecutive sets performed with 15RM load during multiple resistance training exercises (squat, leg press and knee extension) for front Femoris muscles. It was hypothesized that 1) neither of the RIL1 or RIL2 would promote the sustainability of repetitions, and 2) that the RIL3 would promote the achievement of greater total repetitions.

Methods:

Subjects: Fourteen trained males volunteered to participate in current study. The inclusion criteria for the study were that all participants had to be habitually physically active, having consistently performed multiple sets for front Femoris muscles at least three times per week for the previous year and were not using any supplements. Subjects' mean $( \pm \mathrm{SD})$ age, body weight and height were $25.6 \pm 4.1 \mathrm{yr}, 76.8 \pm 7.1 \mathrm{~kg}$, $174.2 \pm 2.43 \mathrm{~cm}$, respectively. All subjects were examined by a physician, and none had any medical problems. Each subject was provided with an information sheet setting out details of the experiment and completed a medical history questionnaire before providing written informed consent. Training experience and habitually physical activity were determined by using questionnaire and interview. Subjects were allowed to resume their normal lifestyle, maintain their normal food intake and hydration, and continue with their normal workouts with avoiding any form of strength exercise for front Femoris muscles in their personal workouts throughout the duration of the study.

\section{Experimental design}

Over a period of 3 weeks, data collection was 
occurred with one experimental testing session per week. During the 3 testing sessions, repetitions to voluntary exhaustion of squat, leg press, and knee extension over 5 consecutive sets with a 15 repetition maximum (15RM) -

procedures were used to determine the order of exercises and the rest interval between sets and exercises during each testing session.

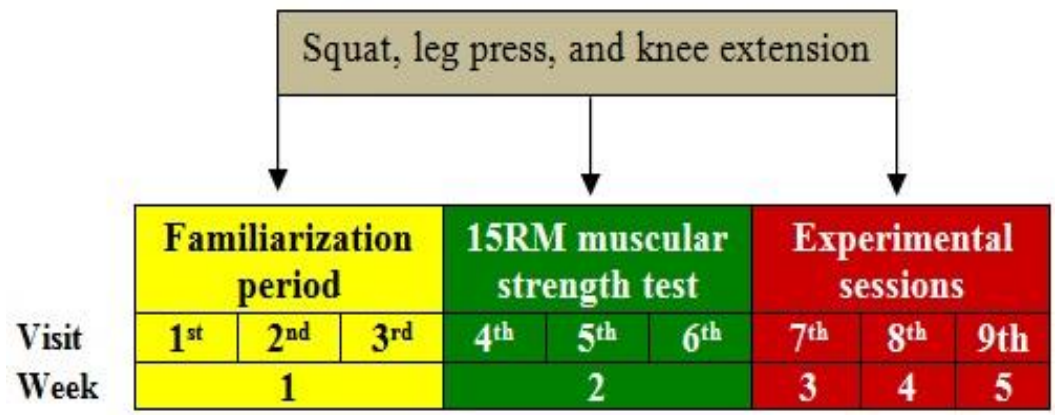

Fig1. Experimental design

Two weeks before the start of experiment, all subjects report to the gym 6 times in non-consecutive days. On the $1^{\text {st }}$ visit; all subjects were examined by a physician and a medical history questionnaire was completed and signed, they changed into sports clothes (running shoes, shorts and t-shirt) height and body weight were recorded using a portable stadiometer and balance weighing scales respectively. On the next 2 visits; all subjects completed 2 load were performed. The 3 testing sessions differed only in the length of rest interval [1min (RIL1), 2-min (RIL2), or 3-minute (RIL3)] between sets and exercises. Counterbalanced and randomized 
set manner on 3 separate days, with 48- 72 hour between them. A high interclass correlation was found between the $2^{\text {nd }}$ and $3^{\text {rd }} 15 \mathrm{RM}$ trails $(\mathrm{R}=$ 0.972). The greatest load lifted over the last two trails was considered the 15RM value. Before the test the subjects performed a warm-up which included $5 \mathrm{~min}$ of light treadmill running and $5 \mathrm{~min}$ stretching exercises. Thereafter, the subjects performed 10 repetitions at a relatively light load that served as a specific warm-up, followed by a gradual increase in load until 15RM was achieved (W. J. Kraemer, Fry, Ratamess, \& French, 1995). During the test, all subjects received verbal encouragement and the exercise technique was monitored and corrected as needed. The rate of the gradual increase in load was dependent on the participant's selfperceived capacity, and it ranged from 5 to $10 \mathrm{~kg}$ for all exercises, with $10 \mathrm{~min}$ rest interval between attempts. The $15 \mathrm{RM}$ was achieved within 3-5 attempts.

\section{Experimental sessions}

Three to four days after the determination of optimum loads for $15 \mathrm{RM}$, all subjects took part in 3 experimental sessions $\quad(1$ experimental session per week). On the day of experimental session, the subject attended to the gem, changed into sports clothes, and performed a warm-up which included 3 min of light treadmill running and $5 \mathrm{~min}$ stretching exercises. Thereafter, the subjects performed 12 repetitions with $40 \%$ of 15RM for each exercise with $3 \mathrm{~min}$ rest between exercises. After a 5 min rest, all subjects performed 5 sets with 15RM load to voluntary exhaustion of squat, leg press, and knee extension (in the same sequence). The 3 experimental sessions differed only in the length of rest interval between sets and exercises RIL1, RIL2, or RIL3 with a random and counterbalance order to experimental sessions. The subjects were verbally encouraged to perform all sets to exhaustion. No attempt was made to control the repetition velocity; however, the subjects were required to utilize a smooth and controlled motion with no pause between the concentric and eccentric phase and between repetitions. The experimental sessions took 
place in June. To minimize circadian variations in muscle strength, all subjects performed their sessions at the same hour of the day (from 7:00 to 9:00 a.m.). The sustainability of repetitions during resistance training was assessed via the recording the number of repetitions in each set, and total repetitions for each experimental session. The repetitions in the first set of each experimental session were expressed as a percentage of the repetition target $(15 \mathrm{RM})$ : (number of repetitions of $1 \mathrm{st}$ set $x$ 100) / 15. In order to calculate the percentage maintained in the number of repetitions relative to the first set (i.e., sustainability of repetitions), the following equation was used: (number of repetitions in $2^{\text {nd }}$ or $3^{\text {rd }}$ or $4^{\text {th }}$ or $5^{\text {th }}$ set $x$ 100) / number of repetitions in the $1^{\text {st }}$ set (Jambassi Filho et al., 2012). The total repetition of each experimental session was calculated by summing the number of repetitions in all five sets.

\section{Statistical analysis}

All data are presented as mean \pm and standard deviations (SD). The statistical calculations were performed using STATISTICA software version 10 (StatSoft, Tulsa, OK, USA). All variables presented normal distribution and homoscedasticity. The sustainability of repetitions was evaluated separately for the squat, leg press, and knee extension, so the repeatedmeasures analysis of variation (ANOVA) (one group, 3 conditions $\mathrm{X} 5$ levels) was used, and when statistical significance $(P<0.05)$ was found, the Tukey HSD post hoc test for comparisons was applied to compare the sustainability of repetitions between sets and between rest conditions. Test-retest reliability for 15RM was determined using an interclass correlation coefficient (ICC).

\section{Results:}

Squat; the $3 \times 5$ repeated ANOVA indicated a significant intra-subject effect for sets $\left(\mathrm{F}_{(1,16)}=173, p=\right.$ $0.000)$ and a significant intersubject effect for rest $\left(\mathrm{F}_{(1,16)}=\right.$ $94.48, p=0.000)$. Post hocs for sets indicated a significant decrease in the repetitions completed between the $1^{\text {st }}$ and each subsequent set thereafter in all RIL (Table 1), (Figure 1). Post hocs for rest indicated a significant difference in the 
sustainability of repetitions between RIL1 and RIL3 and between RIL2 and RIL3. However, the sustainability of repetitions was not significantly different between RIL1 and RIL2 (Table 2), (Figure 2).

\section{Table (1)}

Mean and standard deviation values for repetitions completed.

\begin{tabular}{|c|c|c|c|c|c|c|}
\hline Exercise & ond & 1 & et 2 & Set 3 & Set 4 & Set 5 \\
\hline $\begin{array}{l}\overline{\underline{\pi}} \\
\stackrel{\vec{\sigma}}{\sigma}\end{array}$ & $\begin{array}{l}\text { RIL1 } \\
\text { RIL2 } \\
\text { RIL3 }\end{array}$ & $\begin{array}{l}15.14 \\
(0.37) \\
15.07 \\
(0.27) \\
15.14 \\
(0.36) \\
\end{array}$ & $\begin{array}{c}13.07 \\
(0.62)^{*} \\
13.14 \\
(0.66)^{*} \\
14.63 \\
(0.50)^{*}\end{array}$ & $\begin{array}{c}11.50 \\
(0.52) * \$ \\
11.71 \\
(0.83) * \$ \\
13.07 \\
(0.47) * \$\end{array}$ & $\begin{array}{c}10.43 \\
(0.51)^{*} \& \& \\
10.79 \\
(0.58) * \$ \& \\
12.21 \\
(0.80)^{*} \$ \&\end{array}$ & $\begin{array}{c}9 \\
(0.67) * \$ \& \# \\
9.36 \\
(0.50)^{*} \$ \& \# \\
11 \\
(0.68) * \$ \& \#\end{array}$ \\
\hline 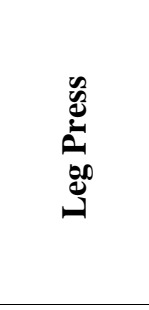 & $\begin{array}{l}\text { RIL1 } \\
\text { RIL2 } \\
\text { RIL3 }\end{array}$ & $\begin{array}{l}11.43 \\
(0.51) \\
11.71 \\
(0.47) \\
12.64 \\
(0.50) \\
\end{array}$ & $\begin{array}{c}9.64 \\
(0.50)^{*} \\
10.07 \\
(0.47)^{*} \\
11.64 \\
(0.63)^{*}\end{array}$ & $\begin{array}{c}8.21 \\
(0.85) * \$ \\
8.71 \\
(0.83) * \$ \\
10.64 \\
(0.74) * \$\end{array}$ & $\begin{array}{c}7.28 \\
(0.47) * \$ \& \\
7.57 \\
(0.65) * \$ \& \\
9.57 \\
(0.64) * \$ \&\end{array}$ & $\begin{array}{c}6.43( \\
0.51) * \$ \& \# \\
6.71 \\
(0.61) * \$ \& \# \\
8.92 \\
(0.47) * \$ \&\end{array}$ \\
\hline 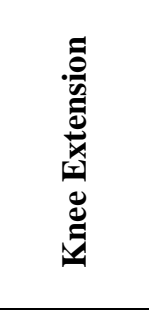 & $\begin{array}{l}\text { RIL1 } \\
\text { RIL2 } \\
\text { RIL3 }\end{array}$ & $\begin{array}{l}10.14 \\
(0.36) \\
10.42 \\
(0.51) \\
11.28 \\
(0.61)\end{array}$ & $\begin{array}{c}8.79 \\
(0.70)^{*} \\
9.14 \\
(0.77)^{*} \\
10.28 \\
(0.61)^{*}\end{array}$ & $\begin{array}{c}7.36 \\
(0.63) * \$ \\
7.64 \\
(0.64) * \$ \\
9.21 \\
(0.58) * \$\end{array}$ & $\begin{array}{c}5.79 \\
(0.58)^{*} \$ \& \\
6 \\
(0.78)^{*} \$ \& \\
8.14 \\
(0.66)^{*} \$ \&\end{array}$ & $\begin{array}{c}4.14 \\
(0.36) * \$ \& \# \\
4.36 \\
(0.50)^{*} \$ \& \# \\
7.07 \\
(0.73) * \$ \& \#\end{array}$ \\
\hline
\end{tabular}

RIL1-rest interval length $1 \mathrm{~min}$, RIL2-rest interval length $2 \mathrm{~min}$, RIL3-rest interval length $3 \mathrm{~min}$, *statistically significant difference in comparison with set1, \$statistically significant difference in comparison with set2, \&statistically significant difference in comparison with set3, \#statistically significant difference in comparison with set4 $(\mathrm{p}<0.05)$. 


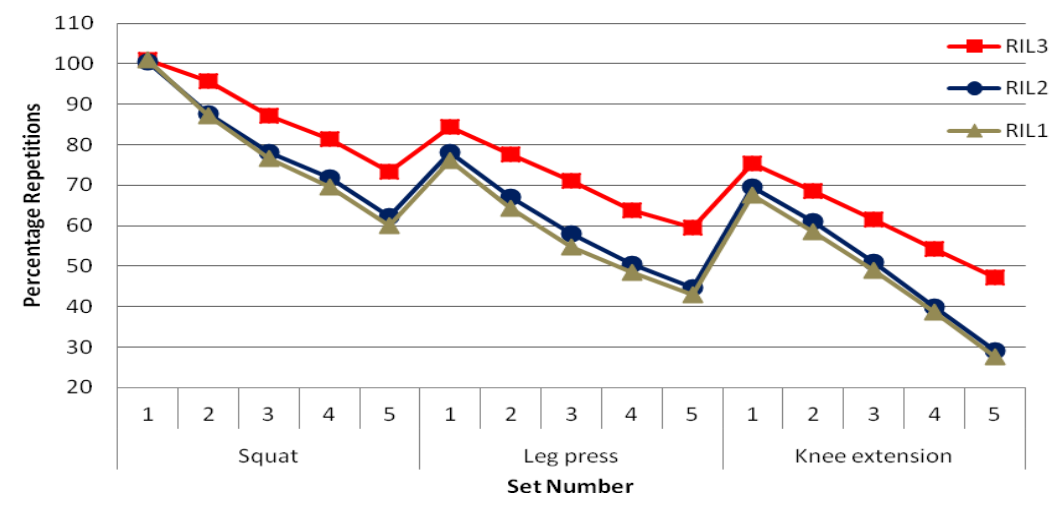

Fig 1. Percentage decline in repetitions per set of Squat, leg press, and knee extension.

Leg Press; the 3 X 5 repeated ANOVA indicated a significant intra-subject effect for sets $(\mathrm{F}$ $(1,16)=498.60, \mathrm{p}=0.000)$ and $\mathrm{a}$ significant inter-subject effect for rest $\left(\mathrm{F}_{(1,16)}=112.11, \mathrm{p}=\right.$ 0.000). Post hocs for sets indicated a significant decrease in the repetitions completed between the $1^{\text {st }}$ and each subsequent set thereafter in all

\section{Table (2)}

RIL (Table 1), (Figure 1). Post hocs for rest indicated a significant difference in the sustainability of repetitions between RIL1 and RIL3 and between RIL2 and RIL3. However, the sustainability of repetitions was not significantly different between RIL1 and RIL2 (Table 2), (Figure 2).

\section{Mean and standard deviation values for total repetitions} completed.

\begin{tabular}{|c|c|c|c|c|}
\hline $\begin{array}{l}\text { Rest Interval } \\
\text { Length }\end{array}$ & Squat & Leg Press & $\begin{array}{c}\text { Knee } \\
\text { Extension }\end{array}$ & $\begin{array}{c}\text { Total } \\
\text { Exercises }\end{array}$ \\
\hline $\begin{array}{l}1 \text { min } \\
2 \text { min } \\
3 \text { min }\end{array}$ & $\begin{array}{c}59.14(2.38) \\
60.07(2.20) \\
65.79(1.61) \\
€ ¥\end{array}$ & $\begin{array}{c}43(1.98) \\
44.79(1.99) \\
53.43(1.51) \\
€ ¥ \\
\end{array}$ & $\begin{array}{c}36.21(2.37) \\
37.57(2.42) \\
46(1.67) € ¥\end{array}$ & $\begin{array}{c}138.35 \\
142.43 \\
165.22 € ¥\end{array}$ \\
\hline \multicolumn{5}{|c|}{$\begin{array}{l}€ \text { Statistically significant } \\
\text { difference in comparison with } \\
\text { RIL1, ¥statistically significant }\end{array}$} \\
\hline \multicolumn{3}{|c|}{ Assiut Journal For Sport Science Arts } & & \\
\hline
\end{tabular}




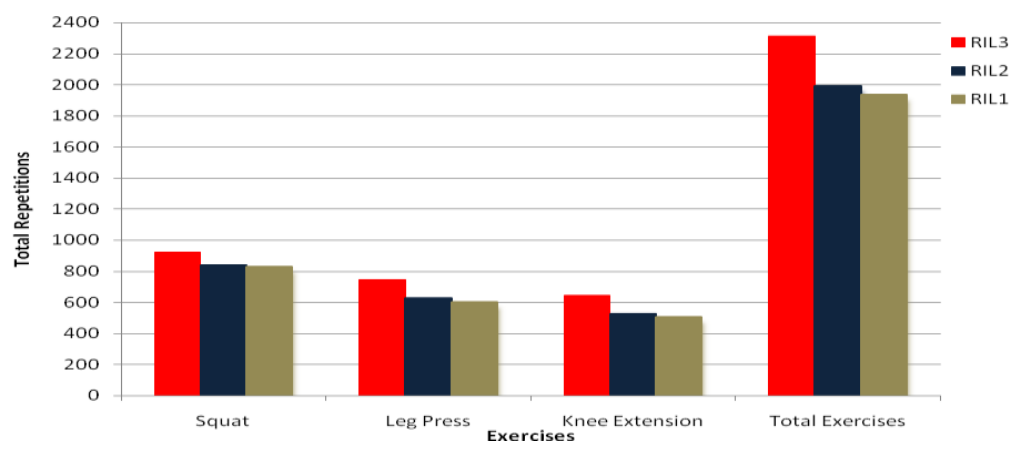

Fig 2. Total repetitions of squat, leg press, and knee extension and total exercises.

Knee Extension; the 3

X 5 repeated ANOVA indicated a significant intrasubject effect for sets $\left(\mathrm{F}_{(1,16)}=\right.$ 769.88, $\mathrm{p}=0.000$ ) and $\mathrm{a}$ significant inter-subject effect for rest $\left(\mathrm{F}_{(1,16)}=98.07, \mathrm{p}=\right.$ 0.000). Post hocs for sets indicated a significant decrease in the repetitions completed between the $1^{\text {st }}$ and each subsequent set thereafter in all RIL (Table 1), (Figure 1). Post hocs for rest indicated a significant difference in the sustainability of repetitions between RIL1 and RIL3 and between RIL2 and RIL3. However, the sustainability of repetitions was not significantly different between RIL1 and RIL2 (Table 2), (Figure 2).

Discussion:
The rest interval between sets is an important variable that affects both acute responses and chronic adaptations to resistance exercise programs. The previous findings recommended that if more than 2 to 3 sets of an exercise are performed, then at least 2 minutes of rest might be needed to minimize load reductions and maintain repetition performance for the sets performed at the end of a workout (De Salles et al., 2009). The results of the current study demonstrated that repetitions were not sustainable over 5 consecutive sets performed with a $15 \mathrm{RM}$ load, irrespective of the rest condition (Table 1, Figure 1). Other studies, which examined the effect of different rest intervals on the sustainability of muscular performance, have demonstrated mixed results. Weir et al. (1994) and 
Matuszak et al. (2003) demonstrated that a maximal squat and bench press were repeatable following a $1,3,5$, or $10 \mathrm{~min}$ rest interval. However, a limitation of these studies was the performance of only two 1RM sets. Therefore, the results of these studies might be applicable to strength testing, but not to actual resistance training workouts, which involve a higher volume of training. Miranda et al. (2007) found similar results to the present study when comparing the effects of one and three-minute rest intervals on the number of repetitions per sets, total volume of each exercise. In the abovementioned study, 14 trained men performed two training sessions, consisting of three sets with 8RM loads, in six exercises for upper body, in the following order: lat pull-down with a wide grip, lat pull-down with a close grip, machine seated row, barbell row lying on a bench, dumbbell seated arm curl and machine seated arm curl. The two experimental sessions differed only in the rest interval between sets and exercises (one and threeminute). For all the exercises, the results showed a lower total number of repetitions when one-minute intervals were used. Both protocols resulted in significant reductions in the third set compared with the first set, in four of the six exercises. Moreover, the protocol that used one-minute also showed reductions in the second set compared with the first set, in two of the six exercises. Although using different intervals and exercises, the results of this study are similar to ours, by showing that shorter rest intervals between sets and exercises can result in declines on the total repetitions of each exercise, significantly greater repetitions were completed for all exercises when resting three minutes between sets, and showed that longer intervals allow the sustainability of the number of repetitions in sets and subsequent exercises performed in a same training session. Additionally, the results of Miranda et al. (2007) showed tendencies towards more substantial reductions in the number of repetitions of the exercises performed at the end of the sequences, which can also be observed in our results.

The main finding of the present study that a 
significantly total repetitions was greater for each exercise when resting 3 minutes between sets and exercises (Table 2) Because the resistance was constant in all five sets of each exercise, these differences in workout volume, due to the greater repetitions completed per set, could be accounted for the 3-minute rest condition (Figure 2). The RIL3 allowed for greater consistency in repetitions over all five sets, whereas the RIL1 and RIL2 did not allow sufficient recovery time. Systematic literature reviews indicate that when multiple sets are performed to voluntary exhaustion, with the maintenance of a constant load throughout all sets, the RI plays a key role in the performance of subsequent sets and in total volume (GarcíaLópez et al., 2008; Miranda et al., 2007; Miranda et al., 2009; Senna, Salles, Prestes, Mello, \& Roberto, 2009). Willardson and Burkett (2005) used a sample of recreationally trained men to determine the effect of 3 different rest intervals on bench press and squat repetitions during a workout. During each testing session, 4 consecutive sets of the squat and the bench press were performed with an 8RM load and a $1 \mathrm{~min}, 2 \mathrm{~min}$, or $5 \mathrm{~min}$ rest interval between sets. For each exercise, the 5-minute rest condition resulted in the highest repetitions completed, followed by the $2 \mathrm{~min}$ and 1 min rest conditions. W. Kraemer, Noble, Clark, and Culver (1987) compared 9 competitive bodybuilders with 8 competitive power lifters on a 10-station resistance exercise circuit. Three consecutive sets of each exercise were performed with a 10RM load that was progressively lowered to allow for 10 repetitions per set. Subjects rested 10 seconds between sets and 30-60 seconds between exercises. The key finding was that the bodybuilders were able to sustain a significantly higher mean percentage of their 1RM during performance of the squat and bench press sets. The authors concluded that the bodybuilders were able to resist fatigue due to adaptations associated with the bodybuilding style of training, which typically consists of moderate to high repetitions with short rest intervals between sets. A key difference between the procedures of (W. Kraemer et al., 1987) and those 
of the current study was lowering the training intensity over consecutive sets. In the current study, the 15RM load was held constant and repetitions were not sustained, irrespective of the rest condition. These results suggest that when training for muscular endurance, the training load should be lowered over consecutive sets to sustain repetitions within the range conducive to this training goal. Based on the results of previous research and those of the current study, the sustainability of repetitions over consecutive sets appear to depend on the magnitude of the load lifted, which in turn effects energy system involvement and the mechanism for fatigue. As training load increases, the body relies increasingly upon intramuscular adenosine triphosphate and phosphocreatine (PCr) stores to supply the energy necessary for muscle contraction (Weiss, 1991). Recovery of PCr stores has been shown to occur relatively quickly, with half of the used $\mathrm{PCr}$ replenished within 30 seconds of recovery (Harris et al., 1976). As demonstrated by Weir et al. (1994) and Matuszak et al. (2003), when training with maximal loads, 1-minute rest intervals hetween repeated attempts were sufficient to recover maximal force production. However, as the load decreases, the body relies increasingly upon anaerobic glycolysis to supply the energy necessary for muscle contraction (Weiss, 1991). Because fast-twitch muscle fibers rely heavily on anaerobic glycolysis for energy production, these fibers accumulate high levels of hydrogen ions during low- to moderate-intensity resistance exercises performed to failure. The accumulation of hydrogen ions has been shown to lower intracellular $\mathrm{pH}$, which results in muscle fatigue (Larson $\mathrm{Jr} \&$ Potteiger, 1997). In other previously conducted experiments of the influence of distinct intervals between sets, the movement velocity of the exercises was controlled (Willardson \& Burkett, 2005, 2006). In our experiment that was not possible, due to the fact that there was a constant decrease in cadence from the first to the last repetition to failure, and one of the indicators of the concentric failure utilized was the reduction in the movement velocity and the consequent pause at concentric phase. Nevertheless, the first repetitions were performed at high velocity and, when fatigue was established, there was a significant decline in velocity, until the exercise was finish. 
This may be a limiting methodological factor of our experiment, since it may affect the number of repetitions, fatigue and type of strength being trained. Our data suggest if sufficient time is available, instituting longer rest intervals (e.g. 3 minutes) allows for a greater number of repetitions and workout volume vs. shorter rest intervals (e.g. $1 \mathrm{~min}$ or 2 min). This performance enhancement has been demonstrated across a wide variety of exercises and muscle groups. So when training for similar muscle groups (Front Femoris Muscles); the rest interval length must be long enough ( $>=3$ minute) to sustain a high number of repetitions over consecutive sets, or decrease the intensity to sustain repetitions.

\section{Conclusion:}

To maintain resistance training goals intensity and volume are important variables to be manipulated. Moreover, the present study showed that the rest interval time between sets and exercises is also a critical variable to improve the same muscle group (front Femoris muscle) using multiple exercises; therefore it directly affects the number of repetitions during the progression of the sets, the total number of repetitions per exercise. These results indicate that these values can be reduced by the use of shorter intervals (RIL1 or RIL2 versus RIL3), and these reductions are less evident during the progression of the sets in the multiple exercises for the same muscle groups. However, there is a great need for further research to compare the volume of work completed over an entire resistance exercise session aimed at training the same muscle groups with different rest intervals between sets.

\section{References:}

Baechle, T. R., \& Earle, R. W. (2000). Essentials of strength training and conditioning: National strength and conditioning association: Human Kinetics.

Campos, G. E., Luecke, T. J., Wendeln, H. K., Toma, K., Hagerman, F. C., Murray, T. F., . . Staron, R. S. (2002). Muscular adaptations in response to three different resistance-training regimens: specificity of repetition maximum training zones. European journal of applied physiology, 88(1-2), 50-60.

De Salles, B. F., Simao, R., Miranda, F., da Silva Novaes, J., Lemos, A., \& Willardson, J. M. (2009). Rest interval between sets in strength training. Sports Medicine, 39(9), 765-777. 
García-López, D., Herrero, J., Abadía, O., García-Isla, F., Uali, I., \& Izquierdo, M. (2008). The role of resting duration in the kinematic pattern of two consecutive bench press sets to failure in elite sprint kayakers. International journal of sports medicine, 29(9), 764-769.

Harris, R., Edwards, R., Hultman, E., Nordesjö, L., Nylind, B., \& Sahlin, K. (1976). The time course of phosphorylcreatine resynthesis during recovery of the quadriceps muscle in man. Pflügers Archiv, 367(2), 137142.

Jambassi Filho, J. C., Gurjão, A. L. D., Ceccato, M., Gonçalves, R., Gallo, L. H., \& Gobbi, S. (2012). Effects of different rest intervals between sets on muscle performance in the leg press exercise in untrained elderly women. Revista Brasileira de Medicina do Esporte, 18(4), 224-228.

Kraemer, W., Noble, B., Clark, M., \& Culver, B. (1987). Physiologic Responses to Heavy-Resistance Exercise with Very Short Rest Periods*. International journal of sports medicine, 8(04), 247-252.

Kraemer, W. J. (1997). A series of studies-the physiological basis for strength training in American football: fact over philosophy. The Journal of Strength \& Conditioning Research, 11(3), 131-142.

Kraemer, W. J., Adams, K., Cafarelli, E., Dudley, G. A., Dooly, C., Feigenbaum, M. S., . . Hoffman, J. R. (2002). American College of Sports Medicine position stand. Progression models in resistance training for healthy adults. Medicine and science in sports and exercise, 34(2), 364380.

Kraemer, W. J., Fry, A., Ratamess, N., \& French, D. (1995). Strength testing: development and evaluation of methodology. Human Kinetics, Champaign, IL, 115-138.

Kraemer, W. J., \& Ratamess, N. A. (2005). Hormonal responses and adaptations to resistance exercise and training. Sports Medicine, 35(4), 339-361.

Larson Jr, G. D., \& Potteiger, J. A. (1997). A comparison of three different rest intervals between multiple squat bouts. The Journal of Strength \& Conditioning Research, 11(2), 115-118.

Matuszak, M., Fry, A. C., Weiss, L. W., Irelamd, T. R., \& 
Mcknight, M. M. (2003). Effect of rest interval length on repeated 1 repetition maximum back squats. The Journal of Strength \& Conditioning Research, 17(4), 634-637.

Miranda, H., Fleck, S. J., Simao, R., Barreto, A. C., Dantas, E. H., \& Novaes, J. (2007). Effect of two different rest period lengths on the number of repetitions performed during resistance training. The Journal of Strength \& Conditioning Research, 21(4), 1032-1036.

Miranda, H., Simão, R., Moreira, L. M., de Souza, R. A., de Souza, J. A. A., de Salles, B. F., \& Willardson, J. (2009). Effect of rest interval length on the volume completed during upper body resistance exercise. Journal of Sports Science and Medicine, 8, 388-392.

Mirzaei, B., Nia, F. R., \& Saberi, Y. (2008). Comparison of 3 different rest intervals on sustainability of squat repetitions with heavy vs. light loads. Brazilian journal of biomotricity, 2(4), 220-229.

Rahimi, R. (2005). Effect of different rest intervals on the exercise volume completed during squat bouts. Journal of
Sports Science and Medicine, 4(4), 361-366.

Ratamess, N. A., Falvo, M. J., Mangine, G. T., Hoffman, J. R., Faigenbaum, A. D., \& Kang, J. (2007). The effect of rest interval length on metabolic responses to the bench press exercise. European journal of applied physiology, 100(1), 1-17.

Rhea, M., Alvar, BA, Burkett, LN, and Ball. (2003). A metaanalysis to determine the dose response for strength development. Med Sci Sports Exerc(35), 456-464.

Richmond, S., \& Godard, M. P. (2004). The effects of varied rest periods between sets to failure using the bench press in recreationally trained men. The Journal of Strength \& Conditioning Research, 18(4), 846-849.

Robinson, J. M., Stone, M. H., Johnson, R. L., Penland, C. M., Warren, B. J., \& Lewis, R. D. (1995). Effects of different weight training exercise/rest intervals on strength, power, and high intensity exercise endurance. The Journal of Strength \& Conditioning Research, 9(4), 216-221.

Sahlin, K., \& Ren, J. (1989). Relationship of contraction capacity to metabolic changes 
during recovery from a fatiguing contraction. Journal of Applied Physiology, 67(2), 648-654.

Senna, G., Salles, B. F., Prestes, J., Mello, R. A., \& Roberto, S. (2009). Influence of two different rest interval lengths in resistance training sessions for upper and lower body. Journal of sports science \& medicine, 8(2), 197.

Todd, J., Sjuts, S., Krosch, B., Conley, D., \& Evetovich, T. (2001). Comparison of varying rest intervals at sixty and ninety percent maximal bench press performance. J. Strength Cond. Res, 15(3), 399.

Weir, J. P., Wagner, L. L., \& Housh, T. J. (1994). The effect of rest interval length on repeated maximal bench presses. The Journal of Strength \& Conditioning Research, 8(1), 58-60.

Weiss, L. W. (1991). The obtuse nature of muscular strength: The contribution of rest to its development and expression. The Journal of Strength \& Conditioning Research, 5(4), 219-227.

Willardson, J. M. (2006). A brief review: factors affecting the length of the rest interval between resistance exercise sets. The Journal of Strength \& Conditioning Research, 20(4), 978-984.

Willardson, J. M., \& Burkett, L. N. (2005). Acomparison of 3Different Rest Intervals on the Exercise Volume Completed During A Workout. The Journal of Strength \& Conditioning Research, 19(1), 23-26.

Willardson, J. M., \& Burkett, L. N. (2006). The effect of rest interval length on the sustainability of squat and bench press repetitions. The Journal of Strength \& Conditioning Research, 20(2), 400-403. 